\title{
The effectiveness and value of aducanumab for Alzheimer's disease
}

\author{
A summary from the Institute for Clinical and Economic Review's California Technology \\ Assessment Forum
}

Patricia G Synnott, MS, MALD; Melanie D Whittington, PhD; Grace A Lin, MD, MAS; David M Rind, MD; and Steven D Pearson, MD

\begin{abstract}
Alzheimer's disease (AD) is a degenerative brain disorder characterized by the progressive loss of memory, cognition, and physical function that affects more than 6 million people in the United States. A hallmark of $\mathrm{AD}$ is the accumulation of beta-amyloid plaques in the brain. In the absence of therapies that alter the course of the disease, treatment for $\mathrm{AD}$ has historically focused on symptom management.
\end{abstract}

On June 7, 2021, aducanumab (Aduhelm, Biogen) became the first potentially disease-modifying therapy to receive regulatory approval from the US Food and Drug Administration (FDA) for treatment of $\mathrm{AD} .^{1} \mathrm{Adu}^{-}$ canumab is a human monoclonal antibody that promotes clearance of beta-amyloid plaques from the brain.

The Institute for Clinical and Economic Review (ICER) evaluated the clinical and economic outcomes of aducanumab for AD. In this review, we present a summary of our findings and highlight key policy recommendations discussed at the California Technology Assessment Forum's public meeting on July 15, 2021. Complete details of ICER's assessment are available at https://icer.org/wp-content/ uploads/2020/10/ICER_ALZ_Final_ Report_080521.pdf. ${ }^{2}$

\section{Summary of Findings}

\section{CLINICAL EFFECTIVENESS}

Aducanumab was evaluated in 2 identical, contemporaneous phase 3 randomized controlled trials, EMERGE and ENGAGE. ${ }^{3}$ The trials randomized 3,285 patients with mild cognitive impairment (MCI) or mild dementia as a result of $\mathrm{AD}$ to low- or high-dose aducanumab or placebo.

In March 2019, EMERGE and ENGAGE were terminated after a prespecified interim futility analysis determined that they were unlikely to reach their primary endpoint. Investigators later examined an expanded dataset that included 3 additional months of data collected between the data cutoff for the futility analysis and the public announcement of termination. The analysis of the larger dataset suggested the possibility of a favorable treatment effect in the EMERGE trial, which formed the basis for the manufacturer's Biologics License Application to the FDA.

Although aducanumab was effective in reducing beta-amyloid plaques in the brain in both trials, clinical outcomes were discordant. The primary endpoint was change in cognition and function, as measured by the 18-point Clinical Dementia Rating Scale-Sum of Boxes (CDR-SB) at week 78. Results from EMERGE suggested a statistically significant slowing of decline in

\author{
Author affiliations \\ Patricia G Synnott, MS, MALD, Center \\ for the Evaluation of Value and Risk in \\ Health, Tufts Medical Center, Boston, \\ MA. Melanie D Whittington, PhD; David \\ $M$ Rind, MD; and Steven D Pearson, \\ $M D$, Institute for Clinical and Economic \\ Review, Boston, MA. Grace A Lin, MD, \\ MAS, Division of General Internal \\ Medicine, University of California, \\ San Francisco. \\ AUTHOR CORRESPONDENCE: \\ Patricia G Synnott, \\ psynnott@tuftsmedicalcenter.org \\ J Manag Care Spec Pharm. \\ 2021;27(11):1613-17 \\ Copyright $@ 2021$, Academy of Managed \\ Care Pharmacy. All rights reserved.
}

the high-dose arm (difference vs placebo $-0.39,95 \% \mathrm{CI}=-0.69$ to -0.09 ), which was less than the 1- to 2-point change that has been suggested as a minimal clinically important difference. ${ }^{3,4}$ Other measures of cognition, function, and behavior also statistically favored high-dose aducanumab in EMERGE. In the ENGAGE trial, however, there were no differences observed between the high-dose aducanumab and placebo arms on 


\begin{tabular}{|c|c|c|c|c|c|c|c|}
\hline Perspective & $\begin{array}{l}\text { Incremental } \\
\text { cost }\end{array}$ & $\begin{array}{l}\text { Incremental } \\
\text { QALYs }\end{array}$ & $\begin{array}{c}\text { Incremental } \\
\text { evLYs }\end{array}$ & $\begin{array}{l}\text { Incremental } \\
\text { life-years }\end{array}$ & $\begin{array}{l}\text { Cost per } \\
\text { QALY }\end{array}$ & $\begin{array}{l}\text { Cost per } \\
\text { evLYG }\end{array}$ & $\begin{array}{l}\text { Cost per } \\
\text { life-year }\end{array}$ \\
\hline \multicolumn{8}{|l|}{ Base cases } \\
\hline Health care system & $\$ 204,000$ & 0.154 & 0.201 & 0.143 & $\$ 1,330,000$ & $\$ 1,020,000$ & $\$ 1,430,000$ \\
\hline Modified societal & $\$ 202,000$ & 0.159 & 0.215 & 0.143 & $\$ 1,270,000$ & $\$ 938,000$ & $\$ 1,420,000$ \\
\hline
\end{tabular}

either the CDR-SB or any other clinical endpoint, similar to previous trials of anti-amyloid drugs. ${ }^{5}$

A primary safety concern in the trials was amyloid-related imaging abnormalities (ARIA) due to edema or effusion (ARIA-E) or brain microhemorrhage or localized superficial siderosis (ARIA-H). ARIA was detected through routine MRI monitoring and was managed through temporary suspension or discontinuation of treatment. Approximately $40 \%$ of patients treated with high-dose aducanumab experienced ARIA, compared with $10 \%$ in the placebo arm. Symptoms included headache, confusion, and dizziness, although nearly $76 \%$ of cases were asymptomatic. Almost all ARIA-E events (98\%) resolved during the treatment period.

\section{LIMITATIONS OF THE CLINICAL EVIDENCE}

It is uncertain why EMERGE and ENGAGE produced inconsistent findings. A key hypothesis for the negative results in ENGAGE was that the timing of a mid-study protocol amendment permitted fewer patients to receive the full high-dose regimen than in EMERGE (22.3\% vs $28.8 \%) .{ }^{3}$ The manufacturer explored this hypothesis extensively in post hoc analyses; however, not all results supported this conclusion. For example, participants who were not affected by the protocol amendment (ie, individuals who were not carriers of the apolipoprotein $\varepsilon 4$ gene) received sustained exposure to high-dose aducanumab yet showed no slowing of decline in either trial.

In addition, the long-term effect of ARIA, including whether there are cumulative effects on the brain, is unknown. This is of particular concern because of the likelihood that patients in routine clinical care will be monitored less frequently. Given the potential for serious adverse effects, it is uncertain whether aducanumab provides sufficient clinical benefit to outweigh its harms.

\section{ECONOMIC EVALUATION}

We developed a Markov model to compare the costeffectiveness of aducanumab plus supportive care with supportive care alone. The analysis used blended efficacy data from EMERGE and ENGAGE to estimate results from both a health care system perspective (ie, focusing on the direct medical care costs and health outcomes of the patient) and a modified societal perspective (ie, including patient productivity impacts, caregiver time, caregiver quality of life, and caregiver direct medical costs). Costs and outcomes were discounted at an annual rate of $3 \%$ over a lifetime time horizon.

The model consisted of 5 health states based on disease severity, including $\mathrm{MCI}$ due to $\mathrm{AD}$, mild $\mathrm{AD}$, moderate $\mathrm{AD}$, severe $\mathrm{AD}$, and death. The model also tracked the setting of care (ie, community or long-term care) in each health state.

Given limitations in the available evidence, the analysis relied on a few key assumptions. First, because our review of the clinical data determined that the findings from EMERGE were no more likely to be "true" than those of ENGAGE, we averaged the results from both trials to estimate the effectiveness of aducanumab for transitions out of the MCI due to AD health state. Second, because the patient population enrolled in the trials was primarily in the MCI health state, transition data out of mild $\mathrm{AD}$ and moderate AD were not available. Following clinical expert consultation, we assumed aducanumab's effectiveness on transitions out of mild $\mathrm{AD}$ to be half of what it was in the MCI health state and assumed no effect on reducing transitions out of the moderate AD health state. Finally, we assumed that patients would discontinue aducanumab treatment once they reached the severe AD health state.

Results from our analysis showed that at an annual list price of $\$ 56,000$ (plus a $6 \%$ markup for administration), incremental cost-effectiveness ratios were $\$ 1.33$ million per quality-adjusted life-year (QALY) gained from the health care system perspective and \$1.27 million per QALY gained from the modified societal perspective. Results between the 2 cost-effectiveness perspectives were nearly identical only because the underlying effectiveness of the drug was quite small. To achieve a cost-per-QALY threshold of $\$ 100,000-\$ 150,000$, the annual price of aducanumab would need to fall within a range of $\$ 2,950-\$ 5,960$. Additional results (incremental cost per life-year and per equal value of life-years gained) are presented in Table 1. 


\section{TABLE 2 Votes on Other Benefits and Contextual Considerations ${ }^{\mathrm{a}}$}

When making judgments of overall long-term value for money, what is the relative priority that should be given to any effective treatment for Alzheimer's disease, on the basis of the following contextual considerations?

\begin{tabular}{l|c|c|c|c}
\hline \multicolumn{1}{|c|}{ Contextual consideration } & $\begin{array}{c}\text { Very low } \\
\text { priority }\end{array}$ & Low priority & $\begin{array}{c}\text { Average } \\
\text { priority }\end{array}$ & $\begin{array}{c}\text { High priority } \\
\text { Hery high } \\
\text { priority }\end{array}$ \\
\hline $\begin{array}{l}\text { Acuity of need for treatment of individual patients based on the } \\
\text { severity of the condition being treated }\end{array}$ & 0 & 0 & 1 & 2 \\
\hline $\begin{array}{l}\text { Magnitude of the lifetime impact on individual patients of the } \\
\text { condition being treated }\end{array}$ & 0 & 0 & 0 & 3 \\
\hline
\end{tabular}

What are the relative effects of aducanumab plus supportive care vs supportive care alone on the following outcomes that inform judgment of the overall long-term value for money of aducanumab?

\begin{tabular}{|c|c|c|c|c|c|}
\hline Potential other benefit or disadvantage & $\begin{array}{l}\text { Major } \\
\text { negative } \\
\text { effect }\end{array}$ & $\begin{array}{l}\text { Minor } \\
\text { negative } \\
\text { effect }\end{array}$ & $\begin{array}{l}\text { No } \\
\text { difference }\end{array}$ & $\begin{array}{l}\text { Minor } \\
\text { positive } \\
\text { effect }\end{array}$ & $\begin{array}{l}\text { Major } \\
\text { positive } \\
\text { effect }\end{array}$ \\
\hline $\begin{array}{l}\text { Patients' ability to achieve major life goals related to education, } \\
\text { work, or family life }\end{array}$ & 2 & 7 & 6 & 0 & 0 \\
\hline $\begin{array}{l}\text { Caregivers' quality of life and/or ability to achieve major life goals } \\
\text { related to education, work, or family life }\end{array}$ & 3 & 7 & 4 & 0 & 0 \\
\hline Society's goal of reducing health inequities & 9 & 4 & 1 & 1 & 0 \\
\hline
\end{tabular}

In a scenario analysis, we used the more favorable data from the EMERGE trial and assumed no reduction in treatment effectiveness in the transition from the mild $\mathrm{AD}$ health state to moderate AD. Even with these more optimistic assumptions, the annual price of aducanumab would still need to be discounted $66 \%-80 \%$ (from $\$ 56,000$ to $\$ 11,000-\$ 19,290)$ to meet a cost-effectiveness threshold range of $\$ 100,000-\$ 150,000$ per QALY. We did not model a scenario using only the ENGAGE trial's data because a cost-effectiveness analysis is not needed for an ineffective and more costly therapy.

ICER's analysis of the potential budget impact of aducanumab suggested that approximately $2.6 \%$ of 1.4 million eligible patients could be treated within 5 years before crossing a budget impact threshold of $\$ 819$ million per year.

\section{LIMITATIONS OF THE ECONOMIC EVALUATION}

The model was limited by considerable uncertainty around aducanumab's safety and effectiveness. In addition, neither trial gives full insight into the relative effectiveness of the drug in later stages of $\mathrm{AD}$, nor can they provide evidence on the clinical outcomes from ARIA over the longer term. Nonetheless, even in the most optimistic scenario analysis, using data only from EMERGE and applying optimistic assumptions about effectiveness in later stages of $\mathrm{AD}$, at the current annual list price of $\$ 56,000$ the drug does not approach traditional thresholds for cost-effectiveness in the US market.

\section{Policy Discussion}

The California Technology Assessment Forum (CTAF) convened on July 15,2021 , to publicly deliberate the clinical and cost-effectiveness evidence on aducanumab. The CTAF is an independent appraisal committee composed of medical evidence experts, including practicing clinicians, methodologists, and patient engagement and advocacy leaders. Their deliberation included input from clinicians and patient representatives with $\mathrm{AD}$ expertise, as well as public comment from drug manufacturers and the public.

Following the discussion, the panel members deliberated on key questions raised by ICER's report. The panel voted unanimously (15-0) that the available evidence was not adequate to demonstrate that aducanumab plus supportive care is superior to supportive care alone.

The CTAF panel also voted on "other potential benefits" and "contextual considerations" as part of a process intended to signal to policymakers whether there are important considerations when making judgments about long-term value for money not adequately captured in analyses of clinical effectiveness and/or cost-effectiveness. The results of these votes are shown in Table 2 . They highlight several factors beyond the results of the cost-effectiveness analysis that the CTAF panel felt were particularly important for judgments of overall long-term value for money. 
The meeting concluded with a policy roundtable in which insurance and manufacturing representatives, clinical experts, and patient representatives discussed how best to apply the evidence and additional considerations into clinical practice and into pricing and insurance coverage policies. The full set of policy recommendations can be found in the Final Evidence Report on the ICER website. ${ }^{2}$ The key policy recommendations are as follows:

- As there is currently a lack of evidence to support that aducanumab is medically necessary, it is not unreasonable for payers to deny coverage of the drug.

- If Medicare chooses to provide coverage following its National Coverage Determination process, it should work with the National Institutes of Health and other research methodology experts to design a rigorous Coverage with Evidence Development program requiring patients be enrolled in a randomized controlled trial or a trial using a rigorous quasi-experimental "waitlist" research design.

- Payers and policymakers should work to achieve more equitable access to current and future therapies by changing benefit designs in Medicare and private insurance to reduce the maximum amount patients must pay out of pocket.

- Given that the FDA approved aducanumab on the basis of amyloid reduction in the brain, it should establish a clear regulatory framework that specifies a threshold range for amyloid clearance that will be considered "reasonably likely" to provide patient benefit.

- Until further data are generated, patient groups, the manufacturer, and clinicians should emphasize the uncertain nature of the evidence and characterize the potential benefits of aducanumab as a slowing of cognitive and functional decline, not as "improvement" or "return of quality of life."

During the policy discussion, clinical experts suggested a wide range of clinical uptake of aducanumab, with the majority suggesting numbers at or above 100,000 patients over 5 years or sooner. ICER therefore issued an "Access and Affordability Alert" in its final report to signal to policymakers that special measures may need to be considered to ensure that the health care system can absorb the added costs of aducanumab over the short term without reducing access inappropriately or contributing to rapid growth in health care insurance costs.

\section{Conclusions}

While the EMERGE trial was the first late-stage clinical trial of drugs targeting removal of amyloid to potentially show clinical efficacy, it is uncertain whether aducanumab works at all or provides sufficient benefits to outweigh its harms. In addition, at current pricing, aducanumab does not meet commonly cited thresholds for cost-effectiveness and is likely to impose significant strain on health system budgets. Given the tremendous unmet needs of patients and families living with $\mathrm{AD}$, clinical societies, policymakers, manufacturers, and patient groups should advocate for fair pricing and for equitable access to all available treatments.

\section{DISCLOSURES}

Funding for this summary was contributed by Arnold Ventures, The Donaghue Foundation, Harvard Pilgrim Health Care, and Kaiser Foundation Health Plan to the Institute for Clinical and Economic Review (ICER), an independent organization that evaluates the evidence on the value of health care interventions.

ICER's annual policy summit is supported by dues from AbbVie, America's Health Insurance Plans, Anthem, Alnylam, AstraZeneca, Biogen, Blue Shield of CA Boehringer-Ingelheim, Cambia Health Services, CVS, Editas, Evolve Pharmacy, Express Scripts, Genentech/Roche, GlaxoSmithKline, Harvard Pilgrim, Health Care Service Corporation, HealthFirst, Health Partners, Humana, Johnson \& Johnson (Janssen), Kaiser Permanente, LEO Pharma, Mallinckrodt, Merck, Novartis, National Pharmaceutical Council, Pfizer, Premera, Prime Therapeutics, Regeneron, Sanofi, Sun Life Financial, uniQure, and United Healthcare.

Whittington, Rind, and Pearson are employed by ICER. Through their affiliated institutions, Synnott and Lin received funding from ICER for the work described in this summary. In addition, Synnott reports support from Biogen for the Tufts Medical Center Cost-Effectiveness Analysis Registry, which is maintained by the Center for the Evaluation of Value and Risk in Health.

\section{REFERENCES}

1. US Food and Drug Administration. FDA grants accelerated approval for Alzheimer's drug [press release]. June 7, 2021. Accessed October 4, 2021. https://www.fda.gov/news-events/ press-announcements/fda-grants-accelerated-approval-alzheimers-drug

2. Lin GA, Whittington M, Synnott PG, et al. Aducanumab for Alzheimer's Disease: effectiveness and value. Final evidence report and meeting summary. Institute for Clinical and Economic Review. August 5, 2021. Accessed October 4, 2021. https://icer.org/wp-content/ uploads/2020/10/ICER ALZ Final Report 080521.pdf 
3. US Food and Drug Administration. Combined FDA and Applicant PCNS Drugs Advisory Committee briefing document. November 6, 2020. Accessed October 4, 2021. https://www.fda.gov/ media/143502/download
4. Andrews JS, Desai U, Kirson NY, Zichlin ML, Ball DE, Matthews BR. Disease severity and minimal clinically important differences in clinical outcome assessments for Alzheimer's disease clinical trials. Alzheimers Dement (N Y). 2019;5:354-63.
5. Ackley SF, Zimmerman SC, Brenowitz WD, et al. Effect of reductions in amyloid levels on cognitive change in randomized trials: instrumental variable meta-analysis. BMJ. 2021;372:n156. 